\title{
New outbursts from GRB 071017 and 1E 1547.0-5408 discovered in an automated search for SGR-like events in the INTEGRAL archive
}

\author{
Martin Topinka*t \\ University College Dublin, School of Physics, UCD, Belfield, Dublin 4, Ireland \\ E-mail: martin.topinkaeucd.ie
}

\author{
Antonio Martin-Carrillo \\ University College Dublin, School of Physics, UCD, Belfield, Dublin 4, Ireland \\ E-mail: antonio.martin-carillo@ucd.ie
}

\section{Seamus Meehan}

University College Dublin, School of Physics, UCD, Belfield, Dublin 4, Ireland

E-mail: seamus.meehanducd.ie

\section{Lorraine Hanlon}

University College Dublin, School of Physics, UCD, Belfield, Dublin 4, Ireland

E-mail: lorraine.hanloneucd.ie

\section{Brian McBreen}

University College Dublin, School of Physics, UCD, Belfield, Dublin 4, Ireland

E-mail: brian.mcbreen@ucd.ie

\begin{abstract}
An offline search for non-triggered flashes in the INTEGRAL IBIS/ISGRI data has been performed. New flaring events detected on a millisecond timescale extend previous observations of sources GRB 071017 and 1E 1547.0-5408 (also known as 1E 1550-5418 or SGR 1550-5418). The characteristics of the major outbursts detected are presented for both sources. The observed behaviour of GRB 071017 casts doubt on its classification as a GRB.
\end{abstract}

8th INTEGRAL Workshop "The Restless Gamma-ray Universe”,

September 27-30, 2010

Dublin Ireland

\footnotetext{
* Speaker.

${ }^{\dagger}$ Please send comments to this author.
} 


\section{Motivation}

Analysis of INTEGRAL IBIS/ISGRI [1], [2], [3] offline data revealed the millisecond flares of SGR J1833-0832, originally detected by Swift [4]. This discovery motivated a search for SGRlike transient events in the IBIS/ISGRI data, which are below the trigger threshold of IBAS [5]. In addition, a comparison between the $T_{90}$ distribution of GRB durations from CGRO BATSE ${ }^{1}$ and IBIS/ISGRI ${ }^{2}$ suggests that IBAS does not generate triggers for events shorter than $\sim 0.4 \mathrm{~s}$, indicating that about 9 undetected and very short GRBs may be present in the INTEGRAL archive (Fig. 1).

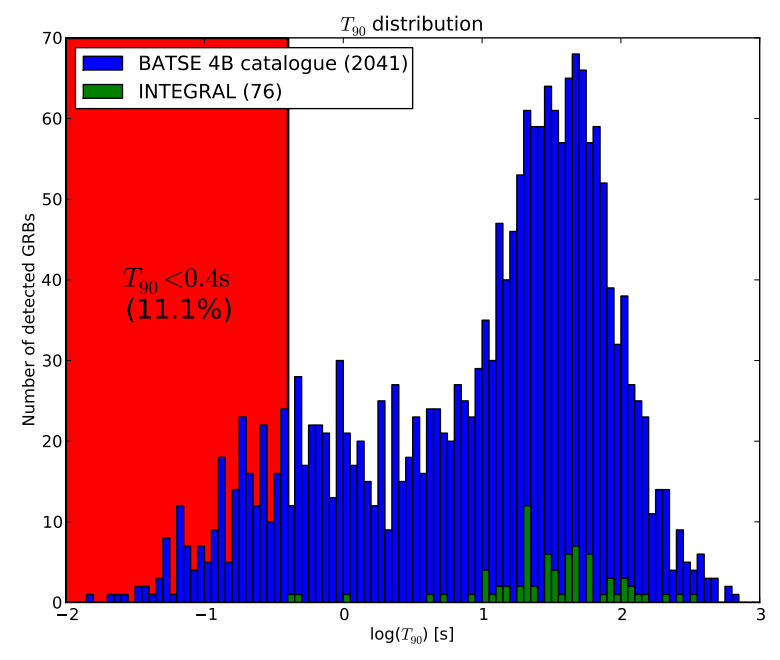

Figure 1: Comparison of the GRB $T_{90}$ duration distributions from IBIS/ISGRI and CGRO BATSE suggesting that there are a number of GRBs not triggered by IBAS (with $T_{90}<0.4 \mathrm{~s}$ ) in the INTEGRAL data.

\section{Peak finding algorithm}

The OSA software cannot detect a new source on a millisecond timescale. Therefore, the event list for an entire Science Window ( $\mathrm{ScW}$ ) was searched for excesses above the real-time background to detect flare candidates which dominate the $\mathrm{ScW}$. The size of the time bin was chosen to contain statistically relevant numbers of counts in each time bin. The data between $20-150 \mathrm{keV}$ were binned into $10 \mathrm{~ms}$ bins and scanned for excesses of more than $10 \sigma$ significance above background, using only good time intervals (GTIs). The software is capable of processing data in both IBIS and SPI event list formats, however, for the purposes of this analysis IBIS data were used exclusively due to the higher sensitivity of the IBIS instrument. The Gaussian nature of the noise (Fig. 2) allows the definition of a threshold criterion for the sum of $n$ successive bins as

$$
\sum_{i} C_{i}+\ldots C_{i+n}-n \mu_{1}>N \sqrt{n} \sigma_{1}
$$

\footnotetext{
${ }^{1}$ http://www.batse.msfc.nasa.gov/batse/

${ }^{2}$ http://isdc.unige.ch
} 
where $C_{i}$ is the number of counts in the i-th bin, $\mu_{1}$ is the average background in 1 bin, $N$ is a threshold and $\sigma_{1}$ is the standard deviation of the noise in 1 bin. To minimize peaks due to random background fluctuations, a 3 -successive-bins $(n=3)$ criterion was used in this analysis. Points above the limit were assumed to be part of a single pulse if they were separated by less than 10 bins.

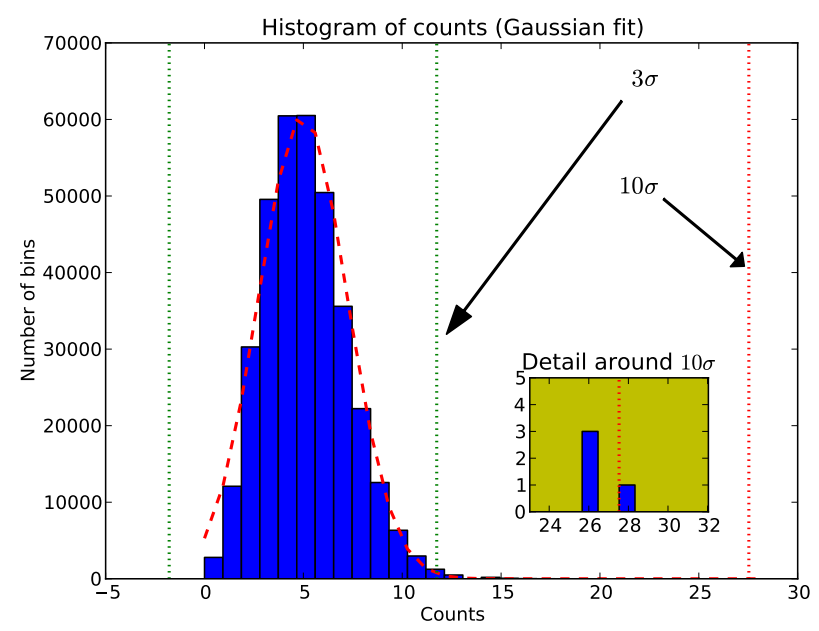

Figure 2: Histogram of IBIS counts for an event list of a random $\mathrm{ScW}$ with $10 \mathrm{~ms}$ binning fit by a Gaussian distribution. The count statistics theoretically follow Poisson statistics. However, the difference between the Poisson and Gaussian distribution is small and since we looked for events above $10 \sigma$, it did not affect the result of the search. The mean value of 5 counts refers to the average background level. The zoomed view shows the excess of counts around $10 \sigma$, suggesting the presence of a flaring source in this ScW.

Events during giant solar flares were automatically excluded in the analysis, since the periods of extreme solar activity are reported within bad time intervals. The software also rejects events caused by cosmic rays. A spike is considered to be the result of a cosmic-ray strike if only a welllocalised region on the detector plane is illuminated rather than a projected shadowgram over the duration of the pulse.

\section{Blind search}

The IBIS archival data was scanned for SGR-like events. There is a total of $60,543 \mathrm{ScWs}$ from $20 / 10 / 2002$ to $30 / 4 / 2009$. Of these, there are 43,332 within $\pm 15^{\circ}$ of the Galactic plane. Not all of the observations are accessible and public. The SGRs are expected to occur close to the Galactic plane. Even when the pointing direction is towards a high galactic latitude, a strong signal through the side of the detector may be obtained. For example, the bright $172 \sigma$ event found within the scans when the instrument was pointing to 3C 279 (Fig. 3), is associated with the outburst of 1E 1547.0-5408 (also known as 1E 1550-5418 or SGR 1550-5418). The next step in this work is to localise the most significant events to establish their source(s).

The average number of events with greater than $10 \sigma$ significance above background in a $\mathrm{ScW}$ is dependent on the Galactic latitude. A typical ScW in the Galactic plane shows 1 event above 


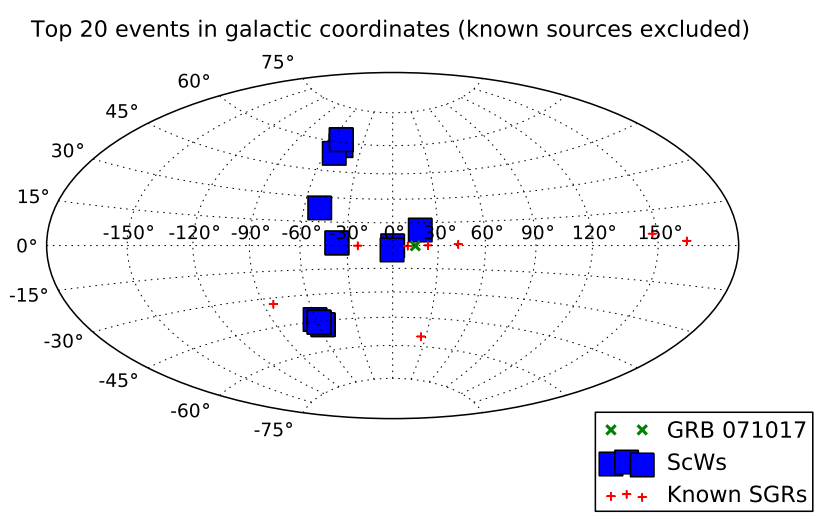

Figure 3: The locations of the ScWs containing the 20 most significant events found within the blind scans. The ScWs in which the known SGRs [6] were within the FOV were excluded. A given ScW may contain multiple 'Top' 20 events. The events in the ScWs around the coordinates $(l=-60, b=60)$ and $(l=-60, b=-45)$ were caused by strong flaring activity of an SGR outside the FOV. The location of the short GRB 071017 is also shown.

$10 \sigma$ and one needs about $10 \mathrm{ScWs}$ to find an event with $25 \sigma$ significance above the background. With increasing latitude, the density of significant events decreases.

\section{Short GRB 071017}

The shortest INTEGRAL GRB to date, GRB 071017 [7], with a $T_{90} \sim 0.4 \mathrm{~s}$ had a spectral power-law index $\sim-3$, that is considerably softer than the majority of short GRBs, and was detected $15^{\prime}$ from the Galactic plane [8]. The main pulse was clearly detected by the pipeline with $16 \sigma$ confidence and the pulse rise matches the reported trigger time (Fig. 4). The source was successfully localised using OSA 5.1 at R.A.18:18:52, Dec. -16:00:03 with an error radius of 2.4'. In addition, the analysis revealed later flaring activity 12 minutes after the GRB pulse with $10 \sigma$ significance and also 18 minutes later with $13 \sigma$ significance. These two outbursts (lasting $0.04 \mathrm{~s}$ and $0.08 \mathrm{~s}$ ) were too weak and short to be localized individually, however when combined into a single image, they are localised within the error circle of the GRB and a spectrum obtained.

The IBIS energy spectrum $(20-100 \mathrm{keV})$ of the combined later pulses yields a power-law of slope $\Gamma=-3.3 \pm 0.3$, consistent with the reported soft spectrum of GRB 071017 itself [8]. The exceptionally long bursting activity, the very soft spectrum and the source location close to the Galactic plane are characteritics more readily associated with SGR-type outbursts rather than a GRB.

\section{1E 1547.0-5408}

1E 1547.0-5408 (also known as 1E 1550-5418 or SGR 1550-5418) went into outburst in Jan- 

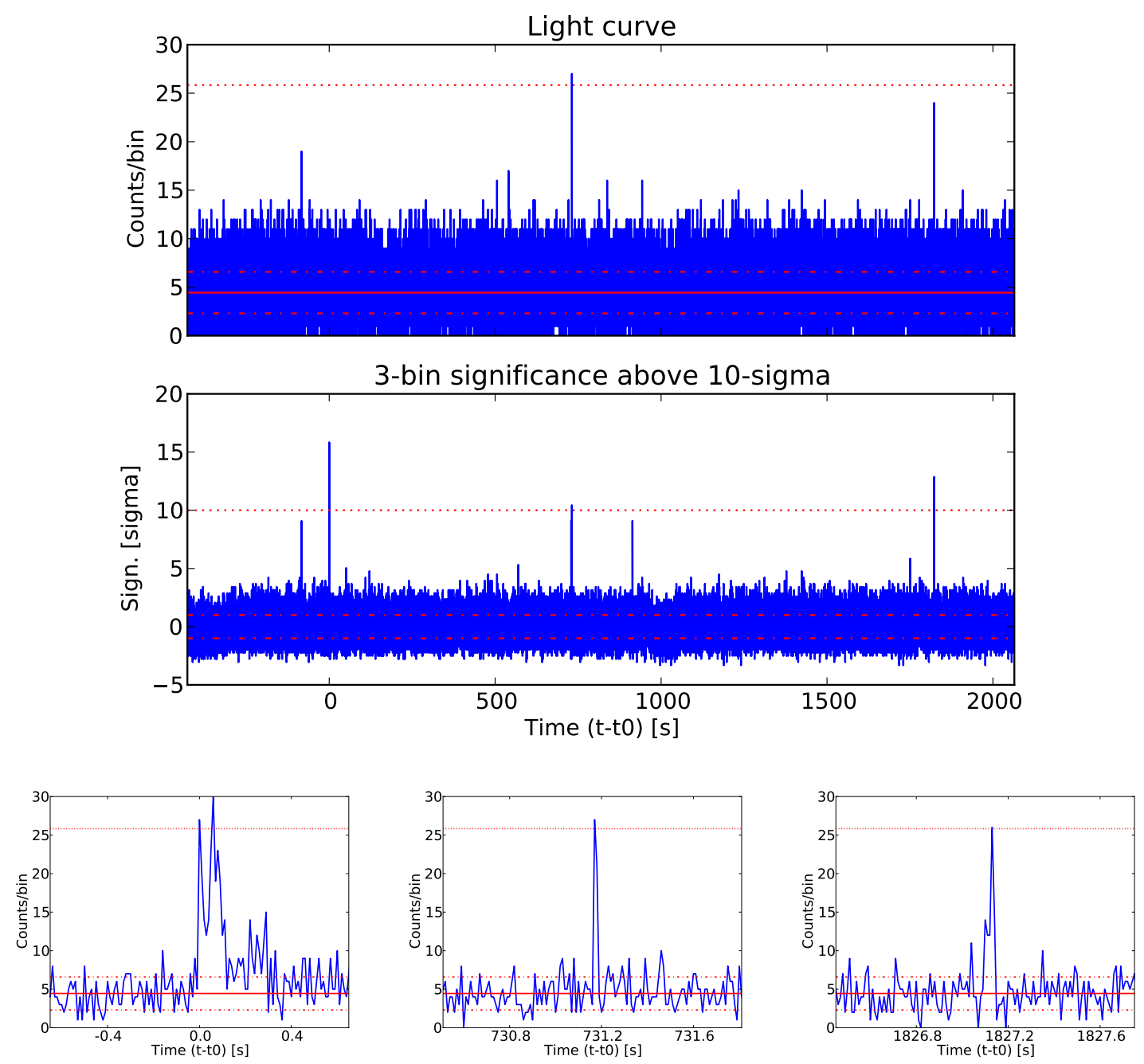

Figure 4: The IBIS light curve $(20-150 \mathrm{keV})$ of the ScW containing GRB 071017 (upper panel) along with the 3-bin significance $(n=3)$ plot (middle panel). The time binning is $10 \mathrm{~ms}$. The horizontal dotted line represents the threshold of $10 \sigma$ in all three subplots. Three flaring events with $>10 \sigma$ significance above background were found. The main outburst of GRB 071017 (the left-most spike, 16 $\sigma$ ) is followed by two other flares shortly after $(11 \sigma$ and $13 \sigma)$. The time profiles of these three events are shown in more detail in the lower panel. $t_{0}$ refers to the burst trigger time on Oct 17, 2007 at 00:58:08.4 UTC.

uary 2009 during which time more than 200 events were detected by Swift and SPI-ACS [9]. The scans have identified an additional 200 flares not reported by other instruments (Table 1), with fluxes of up to $(2.97 \pm 0.05) \times 10^{-4} \mathrm{erg} \mathrm{cm}^{-2} \mathrm{~s}^{-1}(20-200 \mathrm{keV})$. The spectrum of an intense flare $(114 \sigma)$ on Jan $29^{\text {th }}, 2009$, whose localisation is consistent with the known source position, is shown in Fig. 5. 


\begin{tabular}{lccc}
\hline Date period & $\#>10 \sigma$ & \#ScW & Top $[\sigma]$ \\
\hline \hline $24 / 1-25 / 12009$ & 12 & 14 & 27 \\
$28 / 1-2 / 22009$ & 120 & 77 & $129,114,72$ \\
$4 / 2-8 / 22009$ & 103 & 65 & $171,118,102$ \\
\hline
\end{tabular}

Table 1: Summary of the results of the scans performed in the time periods when SPI-ACS and Swift results are missing, or high activity of 1E 1547.0-5408 (also known as 1E 1550-5418 or SGR 1550-5418) was not reported. Col. 2 refers to the number of events above $10 \sigma$ found among the available ScWs, listed in Col. 3 . The significances of the most significant events are listed in Col. 4.

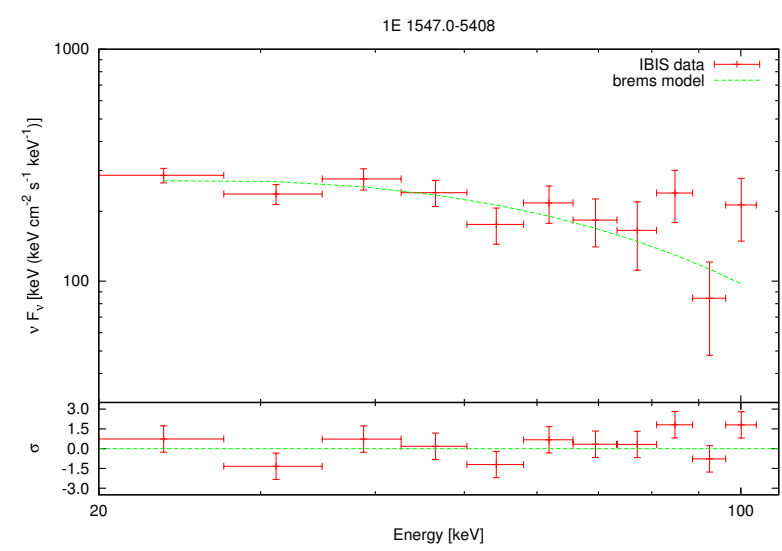

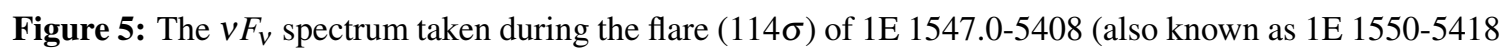
or SGR 1550-5418) on Jan 29th, 2009, beginning at 22:27:33.2 UTC and lasting 0.13 s. The spectrum represents the best fit to a bremsstrahlung model with parameter $k T=43 \pm 4 \mathrm{keV}$. The reduced $\chi^{2}=1.30$ with 9 d.o.f. The fit residuals are shown in the lower panel.

\section{Conclusion}

The archive of the total number of 43,332 IBIS observations was built and the ScWs were scanned for events with $>10 \sigma$ significance above the background. The offline search on the millisecond timescale has revealed new behaviour from the short GRB 071017 and the known 1E 1547.0-5408 (also known as 1E 1550-5418 or SGR 1550-5418). Further analysis of the data is underway.

\section{References}

[1] Winkler, C., et al., 2003, A\&A, 411, L1

[2] Lebrun F. et al., 2003, A\&A, Vol. 411, L141

[3] Ubertini, et al., 2003, A\&A, 411, L131

[4] Kuiper L. and Hermsen W., ATel \#2509, 2010

[5] S. Mereghetti, et al., 2001, Proc. of the 4th INTEGRAL Workshop 459, 513
[6] http://www.physics.mcgill.ca/ $\sim$ pulsar/magnetar/main.html

[7] Mereghetti et al. GCN 6927, 2007

[8] Meehan et al., PoS, p. 47, 2009

[9] Mereghetti et al., 2009, arXiv:0903.1974 